University of Nebraska - Lincoln

DigitalCommons@University of Nebraska - Lincoln

4-6-1994

\title{
Aspect angle estimation of targets in forward looking infrared images using the model-based vision approach
}

Prabir Bhattacharya

Follow this and additional works at: https://digitalcommons.unl.edu/csearticles

This Article is brought to you for free and open access by the Computer Science and Engineering, Department of at DigitalCommons@University of Nebraska - Lincoln. It has been accepted for inclusion in CSE Journal Articles by an authorized administrator of DigitalCommons@University of Nebraska - Lincoln. 


\section{Aspect angle estimation of targets in forward- looking infrared images using the model-based vision approach}

\author{
Prabir Bhattacharya \\ University of Nebraska-Lincoln \\ Department of Computer Science and \\ Engineering \\ Lincoln, Nebraska 68588-0115 \\ E-mail: prabir@cse.unl.edu
}

\begin{abstract}
We develop a model-based vision algorithm to estimate the aspect angle of a target in a forward-looking infrared (FLIR) image. In the preprocessing stage of the algorithm, a set of 3-D voxel-based models is created using a CAD/CAM package. These models are rotated about the vertical axis through a series of predetermined angles and then projected onto the horizontal plane. This gives us a database library of 2-D images. We select as signature from a given FLIR image and attempt to match it with the various images in the given database library of images using the normalized cross-correlation method. The angle of rotation corresponding to the image in the database library giving the best possible match is estimated to be the aspect angle of the signature (target). We use an algebraic approach to represent images and the process involves certain algebraic operations on the polynomials. An advantage of the algebraic approach is that a high speedup in the run time is possible if the fast Fourier transform is used to compute the polynominal multiplications involved in the processing.
\end{abstract}

Subject terms: aspect angle; model-based vision; target geometry; matching; algebraic approach.

Optical Engineering 33(10), 3334-3341 (October 1994).

\section{Introduction}

Automatic target recognition (ATR) is a specialized branch of computer vision that is of considerable interest to the military. Given a set of IR images, the objective of ATR is to recognize a target such as a military vehicle on the ground or a ship in the ocean. The limitations of ATR include the sensor image deficiencies, clutter, topography, vegetation, size of the target, target signature variability, aspect angle dependence, time of the day, weather conditions, etc. In the early 1980s, the Low Altitude Navigation and Targeting Infrared for Night (LANTIRN) program provided an impetus for extensive investigations ${ }^{1-8}$ in ATR. The classical works on ATR used methods of statistical pattern recognitionhere, a geometric view model is built through the estimation of some parameters (such as the sensor elevation angle, aspect angle, the distance from the target, etc.), the key features are extracted from the input image (e.g., by the Hough transform), and recognition/detection is carried out by spatially correlating the features. There has been considerable interest in the integration of thermal and visual images. ${ }^{9}$ For a survey of the applications of the neural network methodology to ATR, see Roth. ${ }^{10}$ For an application of Gabor functions and wavelets to ATR, see, e.g., Casasent, Smokelin, and Ye ${ }^{11}$ and Zhou and Crawshaw. ${ }^{2}$ Several types of sensors are available: the forward-looking infrared (FLIR), the synthetic ap-

Paper 02014 received Jan. 6, 1994; revised manuscript received May 6, 1994; accepted for publication May 6, 1994

(c) 1994 Society of Photo-Optical Instrumentation Engineers. 0091-3286/94/\$6.00. erture radar (SAR), and the multiple-mode laser radar. The use of FLIR images by ATR has been studied extensively in the past 25 years. ${ }^{13-15}$ The interpretation of FLIR images is complex due to various reasons. First, like other types of sensors, we deal with real-time situations where the background and environmental conditions are mostly unknown. Further, as compared to other sensors, the FLIR sensor gives the pilot less information about the target, e.g., the target range cannot be directly computed in a straightforward manner. Also, a FLIR image is often cluttered and it is difficult to perform a good segmentation of the image (independent of conditions such as the time of the day or night when the image was taken, etc.). However, some of the main advantages of the FLIR imagery over other radar images are that the FLIR sensor is widely used for night vision and consequently it is likely to be available to the pilot of a highperformance aircraft, and the FLIR sensor is a passive sensor (i.e., it does not emit any energy of its own) and so it may be more convenient from the operational point of view.

The model-based vision (MBV) approach is a significant method for 3-D object recognition and there has been extensive work on the subject. ${ }^{15-19}$ In the MBV approach, the unknown image is matched with some known models and so can be recognized-it is assumed that the unknown image is approximately similar to one of the models. The MBV approach has important applications in a number of areas such as robotics and industrial inspection. Investigations have been made on the robustness of the MBV approach in cluttered images, e.g., see Vergnet, Saint-Marc, and Ayache. ${ }^{20}$ 
There has been also considerable interest in the application of the MBV approach to ATR and, in particular, to FLIR imagery (e.g., Zelnio ${ }^{7,8}$ and Nasr and Amehdi. ${ }^{21}$ ) The geometry of a FLIR image can be investigated for the purpose of recognition of the target (this is also in general the basic approach in 3-D computer vision ${ }^{22}$ ). There has been considerable interest recently to use the MBV approach to integrate different types of sensors, e.g., a FLASER is a combination of FLIR and SAR sensors. ${ }^{8}$

The aspect angle ${ }^{12,14,24}$ of a target is a parameter that gives information regarding the orientation of a target with respect to a given sensor, and its determination is of significant interest. To explain the definition, consider the target geometry given in Fig. 1 where $O$ represents a sensor stationed above the ground level (e.g., on an aircraft), and $A B$ is a line segment representing the center line of a target (e.g., a vehicle on the ground). Let $C$ denote the midpoint of $A B$, and let the vertical line through $O$ intersect the ground level at $T$ (so $|O T|$ is the height of the sensor above the ground level). The aspect angle of the given target is defined to be the angle $\theta$ between the vertical plane through $A B$ and the plane containing the triangle $O C T$ (we take $0 \leqslant \theta \leqslant \pi$ ). The depression angle $\delta$ of the target is defined to be the $\angle O C T$. The pose of the target is defined to be the information consisting of the depression angle and the aspect angle. It is clear that for low-altitude views the depression angle is relatively insignificant. Because the major objective of the LANTIRN program (mentioned earlier) is low-altitude flying and targeting, it is therefore of considerable practical interest to determine the aspect angle of a target on the ground. Since an infrared image is constrained by several limitations (weather conditions, clutter, etc.), it is more realistic to estimate the aspect angle rather than attempt to determine it absolutely. As different shapes of the target correspond to different aspect angles, the problem of aspect angle estimation tantamounts also to a recognition of the target by attempting to identify it with one of the given models (based on the assumption that the target is approximately identical to one of the models but we do not know which one). A potential application of the aspect angle determination problem also lies in the development of improved sensors and seeker heads for air-to-air missles, e.g., to estimate the maximum angle to which an infrared seeker can possibly be cued.

\subsection{Outline of Proposed Method}

In this paper, we propose a method to estimate the aspect angles of targets in FLIR images using the MBV approach. Our method can also be applied to infrared images from other types of sensors, but it has been developed primarily for the specific capabilities of a FLIR sensor. (We discuss in the following paragraph an alternative method to compute aspect angles and also to explain why that method may not be suitable for FLIR images.) A brief outline of the proposed method is as follows. A set of 3-D models (or military vehicles) is created using the standard BRL/CAD package. We assume that the target (a signature in the given FLIR image) is identical to one of the given models. In the preprocessing stage of the algorithm, each of the given sets of 3-D models is rotated through a series of predetermined angles about the $Z$ axis in the vertical direction. Then, we obtain the projections of these rotated views onto the plane orthogonal to the axis

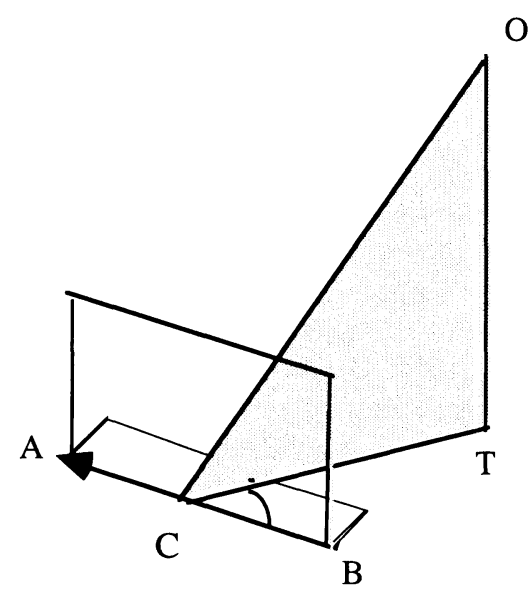

Fig. 1 Definition of the aspect angle of a target.

of rotation (i.e., the $X Y$ plane). We now have a database library of images that can be made as extensive as possibly by choosing more angles of rotation and more models. The FLIR image of the target is now thresholded suitably to obtain a silhouette. In practice, the scene would consist of several signatures, and we could zoom into some specific one whose aspect angle we are interested in determining. We then use a matching algorithm to match the silhouetted image (of the target) with the images in the database library created already, and compute the percentage of matches with each image in the database library. The aspect angle of the target is estimated to be the angle of rotation corresponding to the image in the database library that gives the highest percentage of matches. We could use any standard matching algorithm for binary images. To implement the proposed algorithm, we use an algebraic approach to process images, ${ }^{25-30}$ although the algorithm works if the standard method of representation of images by arrays is used. (The possible advantages of the polynomial representation are explained in Sec. 3.)

\subsection{Main Contributions of the Paper}

Image segmentation is certainly a challenging problem and until now only partial solutions were known to exist. The main contribution of the paper is that we have given an algorithm (undoubtedly straightforward) based on the wellknown MBV approach. To our knowledge, this is the first work based on the MBV approach to estimate the aspect angle or targets from infrared images - a problem of considerable practical interest in automatic target recognition from low altitude flying. Experimental results that we provide indicate clearly that the method gives correct results. We tested the algorithm extensively and the results were always satisfactory. Also, we have indicated (Sec. 5) future directions on how the proposed method can be further refined to give possibly even better results.

We have used an algebraic method to implement the proposed method. The algebraic method is not necessary to implement the proposed algorithm but there are certain advantages if one does so. The possible advantages of the algebraic approach over the standard representation of images has been discussed exhaustively in our previous works. ${ }^{25,26,29}$ Thus, in this paper, this point is only reviewed briefly (see Sec. 3). Besides the possible increase in speed involved, the algebraic 
approach provides a unified environment for various image processing tasks.

\subsection{Another Method}

Although the aspect angle of a target is a significant parameter in ATR and is widely cited, no published literature seems to exist to address directly the problem of its computation. However, we observe that a method to estimate aspect angles could be extracted using ideas from Kato et al. ${ }^{24}$ as follows. In Fig. 2, we have a target geometry showing the vertical plane containing a sensor $O$ and the center line $A B$ of a target. Let $C$ denote the point of intersection of $A B$ with the vertical line through $O$. Let $|A B|=l,|O A|=a,|O B|=b$, and $|O C|=R$. Let $\phi=\angle A O B$ and $\theta=\angle A C O$, the aspect angle of the target. Then, using elementary plane trigonometry, it is straightforward to show that

$\sin \theta=\frac{a+b}{l} \sin \frac{\phi}{2}, \quad \sin \theta=\frac{a b \sin \phi}{R l}$.

If $a, b, l$, and $\phi$ are given, then $\theta$ can be computed from the left equation in Eq. (1). Similarly, if $R, l, a, b$, and $\phi$ are given, then $\theta$ can be computed from the right equation in Eq. (1). Even though this gives us a direct method to estimate the aspect angle of a target, the main problem is that it is difficult, in practice, to compute the values of $a, b$, and $R$ for FLIR images, although $\phi$ can be computed by counting the pixels in the image. If we use an active sensor such as a laser radar, then it would be straightforward to obtain the values of $a, b$, and $R$. The geometric model given by Fig. 2 was used $^{24}$ in the context of targets that are ships for whom the length-to-height ratios are given. However, we think that detecting a target like a tank from an aircraft is much more complex than detecting a ship in the ocean. The ship would appear to be a long thin strip and the segmentation of the image is easy because the image can be modeled as being two-class - the target being either brighter or darker than the scene. Thus, we contend that this method is not generally suitable for aspect angle determination for signatures in FLIR images of tanks and other military vehicles.

In Sec. 2 we discuss some preliminary material. In Sec. 3 we describe the proposed method. Section 4 contains the

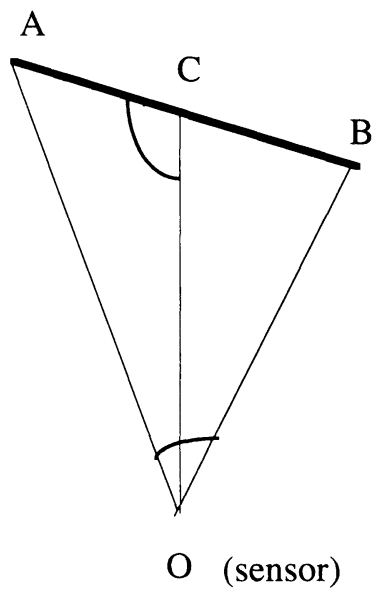

Fig. 2 Target geometry corresponding to Eq. (1). experimental results. Section 5 discusses future directions and Sec. 6 provides the conclusions.

\section{Preliminaries}

To make the paper self-contained, we shall review briefly some facts on the algebraic method used by us for processing images. ${ }^{25-30}$ It is well known that there are essentially three general approaches to represent rigid solids ${ }^{16,17,31}$ - the surface or boundary representation, the generalized cylinders representation, and the volumetric (voxel) representation. Here we use the voxel representation for 3-D images where a solid object is approximated by a volume occupancy array and each cell of the array is called a volume element, or in short, a voxel. Several methods are used to store and process the voxel representation of solids; we consider here an algebraic approach using polynomials. First, given a binary image, say, of size $r \times s$, we associate with it a polynominal in two variables:

$\sum_{i, j} a_{i j} X^{i} Y^{j}$

where the summation is over all the pixels of the image and $a_{i j}$ is 1 or 0 as $(i, j)$ corresponds to a black and white pixel, respectively. Next, a 3 -D object, $A$, given by the voxel representation is encoded by a polynominal $P$ in three variables:

$P \equiv \sum_{i, j, k} p_{i j k} X^{i} Y^{j} Z^{k}$

where $p_{i j k}=1$ if the voxel $(i, j, k)$ of the array lies completely inside $A$, otherwise $p_{i j k}=0$.

\subsection{Rotation}

Our rotation procedure ${ }^{26}$ is an adaptation of the general method of geometric transformation given in Rosenfeld and Kak. ${ }^{32}$ Consider a 3 -D object $S$ with the polynominal representation given by Eq. (3). Let $S^{\prime}$ be the solid obtained by rotating $S$ through an angle $\theta$ about the $O Z$ axis. When $\theta$ is a multiple of $90 \mathrm{deg}$, the polynominal representation of $S^{\prime}$ is easy to obtain from that of $S$. For example, if $\theta=180 \mathrm{deg}$, then we have

$S^{\prime} \equiv \sum_{i, j, k} p_{i j k} X^{-1} Y^{-1} Z^{-1}$

When $\theta$ is not necessarily a multiple of $90 \mathrm{deg}, S^{\prime}$ is obtained as an approximation in the following manner. Suppose that the polynominal representation of $S^{\prime}$ is given by

$\sum_{r, s, t} q_{r s t} X^{r} Y^{s} Z^{t}$

Compute each $(r, s, t)$ from $(i, j, k)$ using the following transformation:

$\left[\begin{array}{l}i \\ j \\ k\end{array}\right]=\left[\begin{array}{lll}\cos \theta & \sin \theta & 0 \\ -\sin \theta & \cos \theta & 0 \\ 0 & 0 & 1\end{array}\right]\left[\begin{array}{l}r \\ s \\ t\end{array}\right]$.

If $\cos \theta$ and $\sin \theta$ are not integers, we round the solution to integral values. For any $r, s, t$ in the set of all real numbers, we get $(i, j, k)$ corresponding to $(r, s, t)$. If $p_{i j k}=1$, then we assign $q_{r s t}=1$, where $(r, s, t)$ is the solution corresponding to 
$(i, j, k)$. We refer the reader to Rosenfeld and $\mathrm{Kak}^{32}$ for details regarding why this indirect method is better than the direct method of computation by the inverse of the transformation given above. In a similar manner, we may obtain rotations about other coordinate axes.

\subsection{Orthographic Projection}

We review briefly the method developed in Qian and Bhattacharya. ${ }^{26}$ Let $P$ be the polynomial representation of a solid $A$ given by Eq. (3). We shall determine the polynominal representations of the orthographic projections of $A$ onto the $X Y$ plane. Let

$Q=\sum_{i, j}\left(\bigvee_{k} p_{i j k}\right) X^{i} Y^{j}$

where $\vee$ denotes the usual Boolean operation OR. We claim that $Q$ is the polynominal representation of the orthographic projection of $A$ onto the $X Y$ plane. For if two or more terms in the polynominal $P$ have the same factor $X^{i} Y^{j}$, then the voxels lying in $A$ corresponding to these terms give the same pixel while projecting orthographically onto the $X Y$ plane. Putting $Z=1$ for these terms in $P$ and then ORing their coefficients gives the projection algebraically. If there is only one term in $P$ with the factor $X^{i} Y^{j}$, then clearly the projection of the corresponding voxel onto the $X Y$ plane is obtained by putting $Z=1$ in the term. The orthographic projections onto the other coordinate planes are obtained in a similar manner.

\section{Proposed Algorithm}

The proposed algorithm for aspect angle estimation has two phases:

Preprocessing. (see Fig. 3)

1. We create a set of models of military vehicles using a CAD/CAM package. The models are stored as 3-D binary, voxel-based images.

2. We rotate each model about the vertical axis ( $Z$ axis) through a series of angles using a rotation algorithm, getting a number of views.

3. We project all the selected views of each model onto the $X Y$ plane using a projection algorithm. We then have a database library of projected views of the given models. Each image in the database has now the following fields: (a) the model from which the image was created and (b) the angle of rotation of the model used to create the image.

\section{Estimation. (see Fig. 4)}

1. Given a FLIR image, we select a certain signature ("target") in it whose aspect angle is to be estimated. A thresholding is now done to obtain a silhouetted figure of the chosen signature.

2. We apply a matching algorithm to match the silhouetted figure with the 2-D images in the database library created in the preprocessing stage.

3. The angle of rotation corresponding to the best match to an image in the given database library is estimated to be the aspect angle of the target.

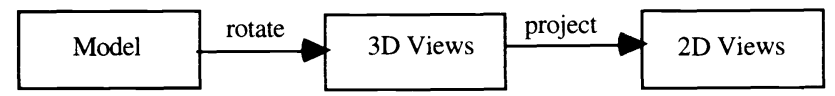

Fig. 3 Block diagram showing the preprocessing phase of the proposed algorithm.

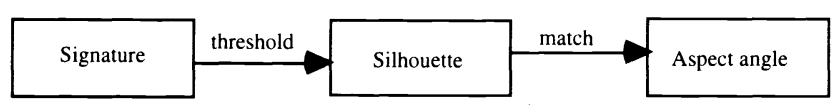

Fig. 4 Block diagram showing the estimation phase of the proposed algorithm.

The estimation of the aspect angle of an unknown target can be made as accurate as possible (subject to various FLIR image deficiencies) by taking as many angles of rotation of the models as we please (and also by taking more models if necessary). Although our proposed method uses simple ideas, it works reasonably well as our experimental results clearly indicate.

In the processing of the images, we shall adopt an algebraic representation where an image (binary, or 3-D voxel-based) is represented by a polynominal and various image processing operations are described in terms of certain algebraic operations on these polynominals representing images. ${ }^{25-30}$ The algebraic operations that we use can be described in a simple manner in terms of the standard operations of addition and multiplication of polynominals. Thus, we get a convenient algebraic environment to process images. Furthermore, if we use the fast Fourier transform (FFT) to compute the multiplications involved in the algebraic operations, then a considerable speedup occurs (specific estimates of the speedup have been given ${ }^{25,26}$ ).

In principle, we need a large database library (of the projections of rotated views of the models), e.g., if there are eight models (of vehicles from tanks to jeeps) and we need to compute the aspect angle up to an accuracy of $1 \mathrm{deg}$, then we need 2880 images in the database library and the matching process with all these 2880 images would be slow. However, in practice, we do not need to estimate the aspect angle correct to the nearest degree - realistically speaking, an estimate correct to, say, $15 \mathrm{deg}$ is acceptable for many purposes. In that if we have, say, eight targets, then the image database library created from the models would have 192 elements.

\section{Experimental Results}

The implementation of the proposed algorithm was carried out using the image processing package KHOROS (developed by the University of New Mexico), which was run on a SUN workstation. We tested the proposed algorithm on a set of simulated FLIR images (supplied by V. Velten of the Wright Laboratory). Each image represented a scene consisting of several M60 tanks together with background clutter and vegetation. Our task was to estimate the aspect angles of each of these tanks correct to $22.5 \mathrm{deg}$. Using the standard BRL/CAD package, a model of an M60 tank was created in the form of a silhouette that was then rotated through steps of $22.5 \mathrm{deg}$ to get 16 templates, which we denote by $t_{i}$ $(0 \leqslant i \leqslant 15)$. From each simulated FLIR image supplied to us, we extracted signatures and matched them, after a suitable 
thresholding, with the 16 templates using the standard normalized cross-correlation method. If the best match occurred with the template $t_{j}$, then the aspect angle of the signature was estimated to be the angle by which the given model had been rotated to obtain $t_{j}$. In general, we found that the results were highly consistent with the actual values of the aspect angles that were made available to us for verification purposes (the file containing the table values of the aspect angles is usually referred to as the "ground truth file.")

Figure 5 shows a simulated FLIR image that has four signatures. To compute the aspect angle of a specific signature in Fig. 5, we do an ad hoc "scissoring" and extract the signature, and then a suitable thresholding. Thus we obtain four pictures that are named Image 1 through Image 4 in a clockwise order according to their positions in Fig. 5, starting with the signature in the bottom left corner. Table 1 gives the sizes of these images and also the thresholding levels that we chose. Table 2 gives the result of matching the images with the templates $t_{i}$ where the second column gives the sizes of the templates $t_{i}$ and the last four columns give the percentages (rounded to two decimal places) of matching the four signatures with the 16 templates. From Table 2, we find that the best match for each image corresponds to $t_{0}$ which is, in fact, the template corresponding to $0 \mathrm{deg}$ rotation. This experimental result agrees with the actual value of the aspect angles as given in the ground truth table.

The percentages of matching of the signatures with the templates $t_{i}$ depend on the value of the thresholding levels chosen. To demonstrate this, we give in Table 3 the percentages of matches of the Image 4 (of Fig. 5) with the templates $t_{i}$ when the thresholding level changes to 167 . Note that the highest percentage of match still corresponds to $t_{0}$, indicating the possible robustness of the proposed algorithm. Similar successful results were also obtained when we tested

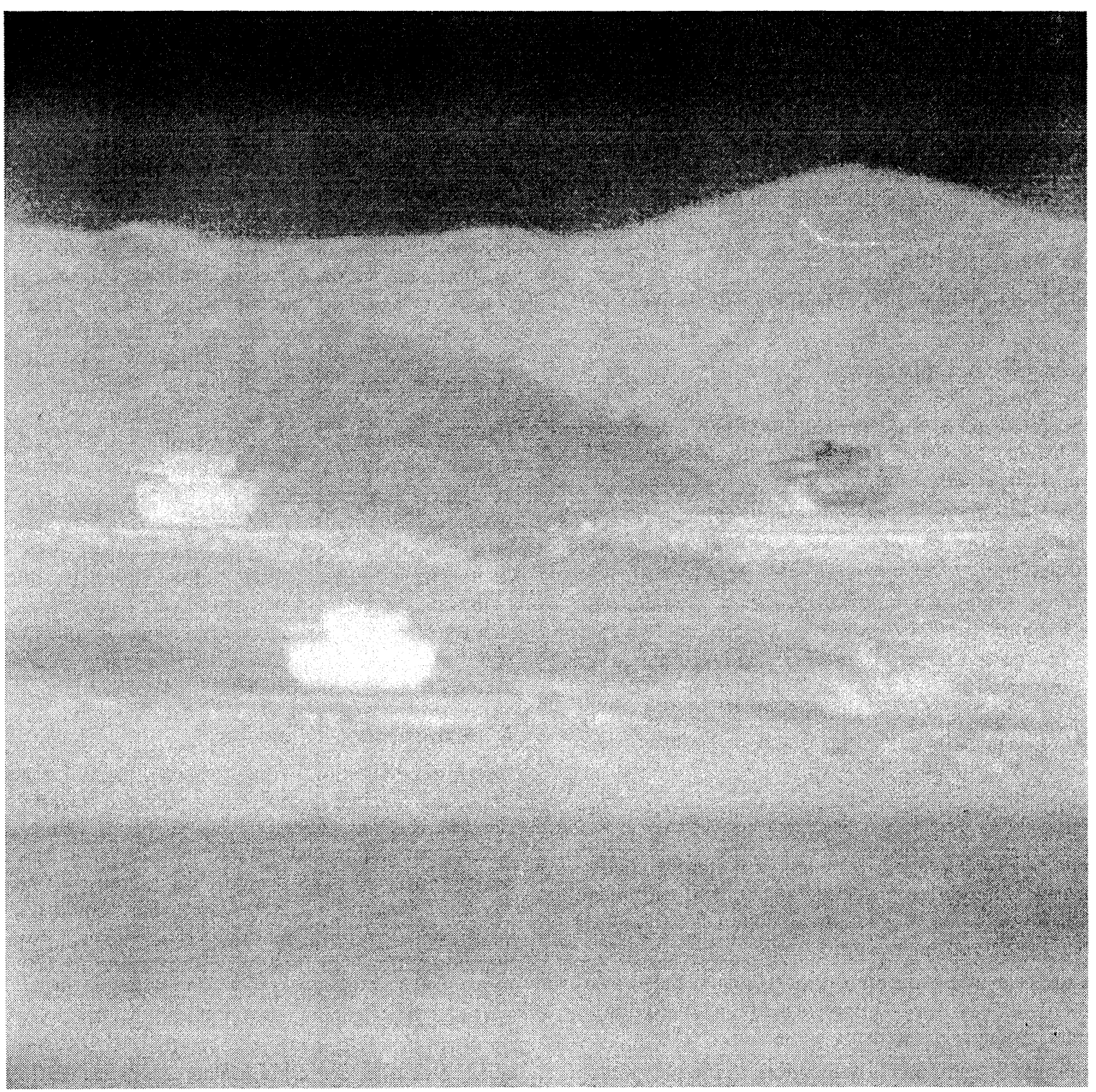

Fig. 5 Image containing signatures whose aspect angles are to be estimated. 
Table 1 Sizes of the signatures in Fig. 5 and their thresholding levels.

\begin{tabular}{|l|l|c|}
\hline Image & Size & Threshold \\
\hline \hline Image 1 & $56 \times 42$ & 182 \\
\hline Image 2 & $56 \times 47$ & 199 \\
\hline Image 3 & $57 \times 76$ & 190 \\
\hline Image 4 & $56 \times 52$ & 173 \\
\hline
\end{tabular}

Table 2 Matching percentages of signatures with the templates.

\begin{tabular}{|c|c|c|c|c|c|}
\hline Template & Size & Image 1 & Image 2 & Image 3 & Image 4 \\
& & Percentage & Percentage & Percentage & Percentage \\
\hline \hline $\mathrm{t}_{0}$ & $70 \times 80$ & 75.51 & 74.67 & 77.49 & 71.65 \\
\hline $\mathrm{t}_{1}$ & $58 \times 76$ & 68.70 & 61.51 & 64.49 & 62.21 \\
\hline $\mathrm{t}_{2}$ & $57 \times 100$ & 64.42 & 57.38 & 60.52 & 58.28 \\
\hline $\mathrm{t}_{3}$ & $61 \times 109$ & 66.76 & 60.99 & 61.82 & 58.17 \\
\hline $\mathrm{t}_{4}$ & $58 \times 102$ & 65.53 & 58.57 & 59.78 & 56.67 \\
\hline $\mathrm{t}_{5}$ & $60 \times 109$ & 65.25 & 58.38 & 61.42 & 57.70 \\
\hline $\mathrm{t}_{6}$ & $57 \times 100$ & 62.25 & 53.69 & 62.97 & 59.94 \\
\hline $\mathrm{t}_{7}$ & $58 \times 76$ & 65.17 & 55.87 & 66.22 & 62.86 \\
\hline $\mathrm{t}_{8}$ & $58 \times 80$ & 48.06 & 43.32 & 44.99 & 41.16 \\
\hline $\mathrm{t}_{9}$ & $58 \times 76$ & 64.89 & 55.65 & 66.28 & 62.75 \\
\hline $\mathrm{t}_{10}$ & $58 \times 76$ & 63.26 & 55.64 & 62.04 & 59.02 \\
\hline $\mathrm{t}_{11}$ & $58 \times 100$ & 43.57 & 42.29 & 34.47 & 32.38 \\
\hline $\mathrm{t}_{12}$ & $59 \times 102$ & 65.44 & 58.93 & 61.28 & 57.90 \\
\hline $\mathrm{t}_{13}$ & $58 \times 109$ & 66.00 & 60.90 & 57.97 & 56.19 \\
\hline $\mathrm{t}_{14}$ & $57 \times 100$ & 65.20 & 58.53 & 60.73 & 58.39 \\
\hline $\mathrm{t}_{15}$ & $58 \times 76$ & 69.55 & 61.93 & 65.47 & 62.38 \\
\hline
\end{tabular}

the proposed algorithm with a number of other images with different aspect angles.

\section{Discussion and Future Directions}

It would be of considerable interest to implement the proposed algorithm on a more complex scene where there may be signatures consisting of a number of military vehicles (from tanks to trucks and jeeps) and with possibly different aspect angles. Then, the implementation of the algorithm would need several models (and their rotated views). The algorithm would first recognize the signature by matching it with various models in the database library, and then estimate the aspect angle by choosing the rotated view with which the highest match was obtained. Thus, the proposed algorithm, in effect, not only estimates the aspect angle but also rec-
Table 3 Matching percentages of Image 4 with the templates when the thresholding level is changed to 167 .

\begin{tabular}{|c|c|}
\hline Template & Match \\
\hline \hline $\mathrm{t}_{0}$ & 63.16 \\
\hline $\mathrm{t}_{1}$ & 59.36 \\
\hline $\mathrm{t}_{2}$ & 55.31 \\
\hline $\mathrm{t}_{3}$ & 57.18 \\
\hline $\mathrm{t}_{4}$ & 55.94 \\
\hline $\mathrm{t}_{5}$ & 55.99 \\
\hline $\mathrm{t}_{6}$ & 52.62 \\
\hline $\mathrm{t}_{7}$ & 54.93 \\
\hline $\mathrm{t}_{8}$ & 42.30 \\
\hline $\mathrm{t}_{9}$ & 54.97 \\
\hline $\mathrm{t}_{10}$ & 53.78 \\
\hline $\mathrm{t}_{11}$ & 37.05 \\
\hline $\mathrm{t}_{12}$ & 55.93 \\
\hline $\mathrm{t}_{13}$ & 57.78 \\
\hline $\mathrm{t}_{14}$ & 55.53 \\
\hline & 59.30 \\
\hline
\end{tabular}

ognizes the signatures as well. We can vary the level of thresholding corresponding to the target and carry out the steps of the algorithm repeatedly until we are able to recognize the test object with a satisfactory level of the matching percentage.

The proposed method may not always give a good output unless an advanced segmentation is used. For this purpose it would be advantageous to use advanced segmentation algorithms. ${ }^{33-36}$ Good segmentation of FLIR images is a challenging problem and it would be interesting to evaluate the test for the performance of the various advanced segmentation algorithms for FLIR images in connection with aspect angle estimation. In our approach, we are matching a silhouette of the signature with a set of rotated views of a number of templates of military vehicles (obtained by the BRL/CAD package). Much work has been done on the problem of shape recognition from single or multiple silhouettes. ${ }^{37-41}$ Adaptation of one of these methods would make the matching algorithm more effective in real-time situations and prevent the occurrence of false alarms as much as possible. Further, it would be useful to create the models as realistically as possible using advanced CAD/CAM techniques so that better matching is obtained.

The proposed algorithm will work whether or not we use the algebraic representation of images (which we have adopted in the implementation). The algebraic representation, however, has certain advantages: It provides a convenient environment to process images ${ }^{25-30}$ where only simple algebraic operations are needed to do the processing of images. 
Also, if we use a FFT package to perform the convolution, which is required in our matching subroutine, we would get a high speed-up of the entire method. So, the database library of the rotated views of the models could be made more extensive without causing a strain on the computing time if we use the FFT to speed up the computing time needed. The algebraic approach is directly amendable to distributed or parallel processing ${ }^{29}$ and it would be useful to extend the proposed algorithm to a parallel algorithm and implement it on a parallel, single instruction multiple data (SIMD) machine.

\section{Summary and Conclusions}

Estimation of aspect angles of targets from IR images is a challenging problem that is of significant interest in the field of ATR. The information about aspect angles is particularly useful for low-altitude flying and targeting using IR images, which is one of the major objectives of the LANTIRN program. A potential application of the estimation problem lies in the task of cueing sensors and seeker heads of missles. We have proposed a MBV method to estimate the aspect angle, which is particularly suitable for FLIR images. Our algorithm involves straightforward ideas but is practical. We create a database library of images from a set of models generated by using a CAD/CAM package and apply a matching algorithm to match a signature with the images in the database library. The confidence level of the proposed method depends on how realistic the models are, the segmentation of the given image, and the number of views of the models. We have used an algebraic environment to represent and process images; this has a number of advantages including the fact that a high speedup of the run time occurs if the standard FFT is used to compute the polynominal multiplications involved. The proposed method to estimate an aspect angle would, however, work without using the algebraic representation of images. The experimental results show good promise of the viability of our method. We have discussed several possible extensions of the proposed method. Since all image processing operations considered here are local operations, the proposed algorithm can be directly extended to a parallel algorithm for an SMID machine. We hope that our work will generate interest in further investigations on the aspect angle estimation problem, and also on further applications of the MBV approach to automatic target recognition.

\section{Acknowledgments}

Part of this work was done during a visit to the MBV Lab of the Wright Laboratories at the Wright-Patterson Air Force Base, Dayton, Ohio in 1992, sponsored by the Summer Faculty Program of the Air Force Office of Scientific Research. I sincerely thank Vincent Velten who hosted my visit and also introduced me to the problem of aspect angle estimation. For the subsequent work, we gratefully acknowledge the support of the Air Force Office of Scientific Research under grant AFOSR F49620-94-1-0029.

\section{References}

1. B. Bhanu, "Automatic target recognition: state of the art survey," IEEE Trans. Aerospace Electron Syst. AES-22(4), 364-379 (1986).

2. W. M. Brown and C. W. Swonger, "A prospect for automatic target recognition," IEEE Trans. Aerospace Electron. Syt. AES-25(3), $401-410$ (1989).
3. P. H. Deitz, M. J. Muuss, and E. O. Davidsson, "Issues in automatic object recognition and material data to predictive signature codes," in Automatic Object Recognition, H. Nass, Ed., SPIE Institute Series IS 7, 40-56 SPIE Press, Bellingham, WA (1990).

4. T. L. Jones, "Development of automatic target recognizers for army applications," Proc. SPIE IS07, 4-13 (1990).

5. J. M. Lloyd, Thermal Imaging Systems, Plenum Press, NY (1975).

6. F. Sadjadi, Ed., Special Section on automatic target recognition,-Opt. Eng. 31(12), 2519-2628 (1992).

7. E. Zelnio, "The importance of sensor models to model-based vision applications,'" in Automatic Object Recgonition, H. Nasr, Ed., SPIE Institute Series IS 7, 98-107 SPIE Press, Bellingham, WA (1990).

8. E. Zelnio, "ATR paradigm comparison with emphasis on model-based vision," in Model-Based Vision Developments and Tools, Proc. SPIE 1609, 2-15 (1991).

9. N. Nandhakumar and J. K. Aggarwal, "Integrated analysis of thermal and visual images for scene interpretation," IEEE Trans. Patt. Anal. Machine Intell. PAMI-10(4), 469-481 (1988).

10. M. W. Roth, "Survey of neural network technology for automatic target recognition," IEEE Trans. Neural Networks 1, 28-43 (1990).

11. D. P. Casasent, J. S. Smokelin, and A. Ye, "Wavelet and Gabor transforms for detection," Opt. Eng. 31(9), 1893-1898 (1992).

12. Y. T. Zhou and R. Crawshaw, "Contrast, size and orientation invariant target detection in infrared imagery,' ' in Automatic Object Recognition, Proc. SPIE 1471, 404-411 (1991).

13. B. J. Shachter, "A survey and evaluation of FLIR target detection/ segmentation algorithms,' Proc. DARPA Image Understanding Workshop, pp. 49-57, Morgan Kaufman, San Mateo, CA (Sep. 1982).

14. D. L. Shumaker, J. T. Wood, and C. R. Thacker, The FLIR Performance Handbook, DCS Corp., Alexandria, VA (1988).

15. N. S. Friedland, "An integrated approach to object recognition," Proc. DARPA Image Understanding Workshop, pp. 777-787 (Apr. 1993).

16. P. J. Besl and R. C. Jain, "Three-dimensional object recognition," $A C M$ Computing Surveys 17, 75-154 (1988).

17. R. T. Chin and C. R. Dyer, "Model based recognition in robot vision," ACM Computing Surveys 18, 67-108 (1986).

18. W. Grimson, Object Recognition by Computer, MIT Press, Cambridge, MA (1991).

19. J. L. Mundy and A. J. Heller, "The evolution and testing of a modelbased object recognition system,"' in Proc. IEEE 3rd Int. Conf. Comput. Vision, pp. 247-261, Osaka, Japan (1990).

20. R. L. Vergnet, P. Saint-Marc, and N. Ayache, "Robustness of modelbased recognition in cluttered images," in Proc. IEEE Comput. Vision Patt. Recog. Conf., pp. 713-714, New York (1993).

21. H. Nasr and H. Amehdi, "Model-based automatic recognition (ATR) development tools," in Automatic Object Recognition, Proc. SPIE 1471, 283-290 (1991).

22. O. Faugeras, Three-Dimensional Computer Vision, MIT Press, Cambridge, MA (1993).

23. J. G. Verly, R. L. Delanoy, and D. E. Dudgeon, "'Model-based system for automatic target recognition from forward-looking laser-radar imagery,"'Opt. Eng. 31(12), 2540-2552 (1992).

24. D. N. Kato, R. D. Holben, A. S. Politopoulos, and B. H. Yin, "Ship classification and aimpoint maintenance," in Infrared Systems and Components, Proc. SPIE 890, 174-181 (1988).

25. P. Bhattacharya, K. Qian, and X. Lu, "An algebraic approach to morphological operations on 2D and 3D images," Patt. Recog. 26, 1785-1796 (1993).

26. K. Qian and P. Bhattacharya, "A 3 D object recognition system using voxel representation," Patt. Recog. Lett. 13, 725-733 (1992).

27. K. Qian and P. Bhattacharya, "Binary image processing by polynominal approach,"' Patt. Recog. Lett. 11, 395-403 (1990).

28. K. Qian and P. Bhattacharya, "An algebraic method for processing colored images,"' Patt. Recog. Lett. 12, 805-811 (1991).

29. K. Qian and P. Bhattacharya, "A template polynominal approach for image processing and visual recognition,' Patt. Recog. 25, 1505-1515 (1992).

30. K. Qian and P. Bhattacharya, "Determining holes and connectivity in binary images," Computers \& Graphics 16, 283-288 (1992).

31. D. Ballard and C. Brown, Computer Vision, Sec. 9.1-9.2, Prentice-Hall, Englewood Cliffs, NJ (1982).

32. A. Rosenfeld and A. C. Kak, Digital Picture Processing, Vol. 2. Sec. 9.4, Academic Press, NY (1982).

33. R. L. Hartley, L. J. Kitchen, C. Y. Wang, and A. Rosenfeld, "Segmentation of FLIR images: a comparative study," IEEE Trans. Syst. Man. Cyber. SMC-12(4), 553-566 (1982).

34. L. Sevigny, G. Hvedstrup-Jensen, M. Bohner, E. Ostevold, S. Ginaker, and J. Dehne, "Discrimination and classification of vehicles in natural scenes from thermal imagery,' Comput. Vision Graphics Image Proc. 24, 229-243 (1983).

35. R. M. Haralick and L. G. Shapiro, "Image segmentation techiques,' Comput. Vision, Graphics, Image Process 29, 100-132 (1985).

36. C. H. Chien and J. K. Aggarwal, "Shape recognition from single silhouette," Proc. IEEE Int. Conf. Comput. Vision, pp. 481-490 (1987).

37. C. C. Chu and J. K. Aggarwal, "The integration of image segmentation 
maps using region and edge information," IEEE Trans. Patt. Anal. Machine Intell. PAMI-15(12), 1241-1252 (Dec. 1993).

38. S. A. Shafer, Shadows and Silhouettes in Computer Vision, Kluwer Academic Publishers, Boston, MA (1985).

39. Y. F. Wang, M. J. Magee, and J. K. Aggarwal, "Matching three-dimensional objects using silhouettes,' IEEE Trans. Patt. Anal. Machine Intell. PAMI-6, 513-518 (1984).

40. R. D. Murch and B. P. McGreggor, "Reconstituting object shape and orientation from silhouettes," J. Opt. Soc. A 9(9) (Sep. 1992).

41. P. L. Van Hove, "Model-based silhouette recognition," Proc. IEEE Comput. Soc. Workshop Comput. Vision, Miami Beach, FL, pp. 88-93 (Dec. 1987).

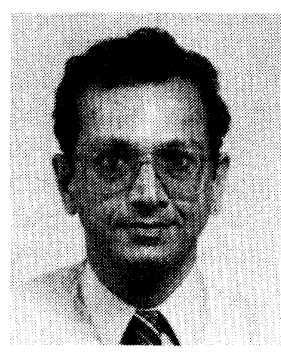

Prabir Bhattacharya received the BA degree (Honors) in 1967 and MA degree in 1970 , both in mathematics from the University of Delhi, India, and a DPhil in 1979 from the University of Oxford, U.K. specializing in group theory. He is currently a professor at the University of NebraskaLincoln, Department of Computer Science and Engineering, that he joined in 1986 as an associate professor. His past assignments included extended visits to the Cen- ter for Automation Research, University of Maryland, College Park; Wright Patterson Air Force Base, Dayton, Ohio; European Molecular Biology Laboratory, Heidelberg, Germany; and the Tata Institute for Fundamental Research, Bombay, India. His current research interests include computer vision, image processing, multidimensional transforms, and parallel computing. He has 50 refereed journal publications, and has coedited one monograph on vision geometry. $\mathrm{He}$ is on the editorial boards of Pattern Recognition, and the Journal of Computing and Information. He is a Fellow of the Institute of Mathematics and its Applications (U.K.), a Chartered Mathematician (U.K.) and a Senior Member of the IEEE. 